Casos Clínicos

Arch. Esp. Urol., 58, 4 (360-362), 2005

\section{CARCINOMA RENAL Y FEOCROMOCITOMA IPSILATERAL}

N uria Rodríguez G arcía, Carlos Pascual M a teo, Luis Lanes $G$ onzález, Carlos Escalera Almendros y Antonio Berenguer Sánchez.

Servicio de Urología. Hospital Universitario de G etafe. $M$ adrid. España

Resumen.- OBJETIVO: presentación de un caso de asociación entre cáncer renal y feocromocitoma ipsilateral, tratado mediante cirugía laparoscópica.

MÉTO DO: describimos el caso de una paciente de 67 años que presenta dolor lumbar izquierdo e infecciones urinarias de repetición. Se practicaron ecografía y CT abdominal, que revelaron carcinoma renal y masa suprarrenal ipsilateral confirmada con RMN . Con diagnóstico de carcinoma renal izquierdo y probable metástasis suprarrenal se realizó nefrectomía y suprarrenalectomía izquierda laparoscópica.

RESULTADO S: el estudio anatomopatológico de la pieza quirúrgica demostró un carcinoma de células renales cromófobo y un feocromocitoma suprarrenal.

CON CLUSIO NES: la asociación de carcinoma renal y feocromocitoma es infrecuente, como así los sugiere la escasa bibliografía al respecto. La presencia de una masa suprarrenal en un paciente con historia de tumor primario suele corresponder frecuentemente a una enfermedad metastásica pero no debe descartarse un proceso neoplásico primario suprarrenal.

Palabras clave: Carcinoma renal. Feocromocitoma.

Summary.- O BJEC TIVES: To report one case of associated renal cancer and ipsilateral pheochromocytoma treated by laparoscopic surgery.

METHODS: We describe the case of a 67 -year-old female presenting with left lumbar pain and recurrent urinary tract infections. Ultrasound and abdominal CT scan were performed revealing renal carcinoma and ipsilateral adrenal mass, which was confirmed by M RI. $W$ ith the diagnosis of left renal carcinoma and possible adrenal metastasis, laparoscopic left nephrectomy and adrenalectomy were performed.

RESULTS: Pathologic study of the specimen reported a chromophobic renal cell carcinoma and adrenal pheochromocytoma.

CON CLUSIO N S: The association of renal carcinoma and pheochromocytoma is unfrequent, as suggested by the limited a vailable bibliography on the topic. The presence of an adrenal mass in a patient with history of primary tumor is frequently secondary to metastasic disease, although primary adrenal neoplasia should not be discarded.

Keywords: Renal carcinoma. Pheochromocytoma

\section{INTRODUCCIÓN}

El feocromocitoma es un tumor poco frecuente, más prevalente en individuos entre 30 y 50 años y más frecuente en mujeres. En algunas familias se hereda de forma autonómica dominante, asociada a síndromes neurectodérmicos, siendo en estos casos su incidencia mayor. Hasta el $17 \%$ de los casos de feocromocitoma pueden ser clínicamente silentes. En el resto, la presentación es en forma de crisis hipertensivas paroxísticas, que se acompañan de cefalea, dolor precordial y ansiedad. Ante la sospecha de este tumor se debe efectuar una determinación de catecola minas en sangre u orina (1). 


\section{CASO CÚNICO}

Mujer de 67 años con antecedentes médicos de hiperlipemia, hipertensión sin tratamiento farmacológico y hernia de hiato. Es remitida a nuestro servicio por dolor lumbar izquierdo e infecciones urinarias de repetición. Se practicó ecografía que evidenció una masa renal izquierda de 3,7 centímetros en polo superior, de características heterogéneas. EI TAC abdominal confirmó una masa renal izquierda compatible con carcinoma renal y lesión nodular suprarrenal ipsilateral. Se realizó RMN para definir mejor las características de la lesión suprarrenal, no cumpliendo ésta las características de adenoma, por lo que se diagnostica como posible metástasis. Se realizó nefrectomía y suprarrenalectomía izquierdas laparoscópica, durante la cual la paciente presentó tensión arterial y frecuencia cardíaca normales. En el postoperatorio inmediato la paciente presentaba tensiones arteriales sistólicas de 150-185 y diastólicas de 75-85 mmmHg.

A los cinco días de la intervención se instauró un cuadro de deterioro del estado general e hipertensión (170/90$190 / 100 \mathrm{mmHg}$ ) refractaria a la administración de enalapril y captopril. Un nuevo TAC abdominal identificó un derrame pleural bilateral sin otros hallazgos de interés. Se pautó tratamiento diurético y antagonistas receptor angiotensina-2 (ARA-2), con lo que se consiguió un buen control de la tensión arterial. Tras este episodio la evolución de la paciente fue satisfactoria.

La anatomía patológica identificó un carcinoma renal tipo cromófobo con extensión a grasa perirrenal $(\mathrm{pT} 3 \mathrm{aN} \times \mathrm{Mx})$ y feocromocitoma en glándula suprarrenal.

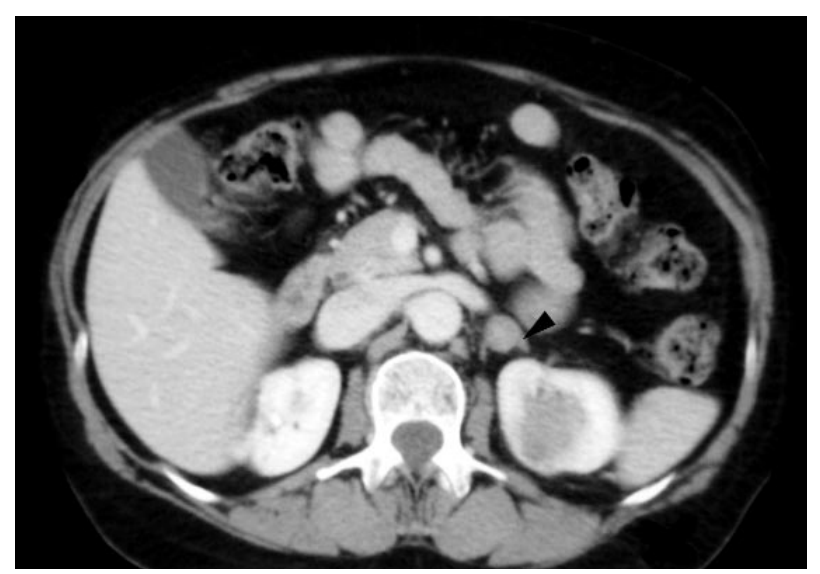

FIG URA 1. CT abdomen: nódulo suprarenal de $1,5 \mathrm{~cm}$ dependiente de rama suprarrenal izquierda

\section{DISCUSIÓN}

Toda masa suprarrenal, aún siendo asintomática, debe ser filiada para descartar que se trate de un tumor funcionante, evitándose así los riesgos que implica una cirugía. En un $4 \%$ de todos los pacientes sometidos a TAC abdominal o torácico se encuentran masas suprarrenales (incidentalomas). En los casos en que ésto constituye un hallazgo en el estudio de una neoplasia recientemente diagnosticada, se asume muchas veces que se trata de lesiones metastásicas. El origen más frecuente de metástasis en las suprarrenales son, por orden de frecuencia: carcinoma renal, melanoma, cáncer colorrectal, pulmón y próstata (2).

A sumir dicha naturaleza metastásica puede implicar llevar a cabo una terapia inapropiada e incluso retrasar el tratamiento del tumor primario.

La asociación de carcinoma renal y feocromocitoma es excepcional; en una revisión bibliográfica de 33 años (1961-2001), se han hallado cinco casos más $(3,4,5)$.

Existen pocos estudios que analicen la evaluación y tratamiento quirúrgico de las masas suprarrenales en pacientes con historia de una enfermedad maligna. Además, en los estudios sobre incidentalomas suelen excluirse los pacientes con neoplasias extra-adrenales sincrónicas o metacrónicas. A sí mismo, en aquellos estudios sobre adrenalectomía se excluyen pacientes con tumores suprarrenales primarios (2).

Por tanto, las metástasis suprarrenales son la causa más frecuente de masa suprarrenal en pacientes con las neo-

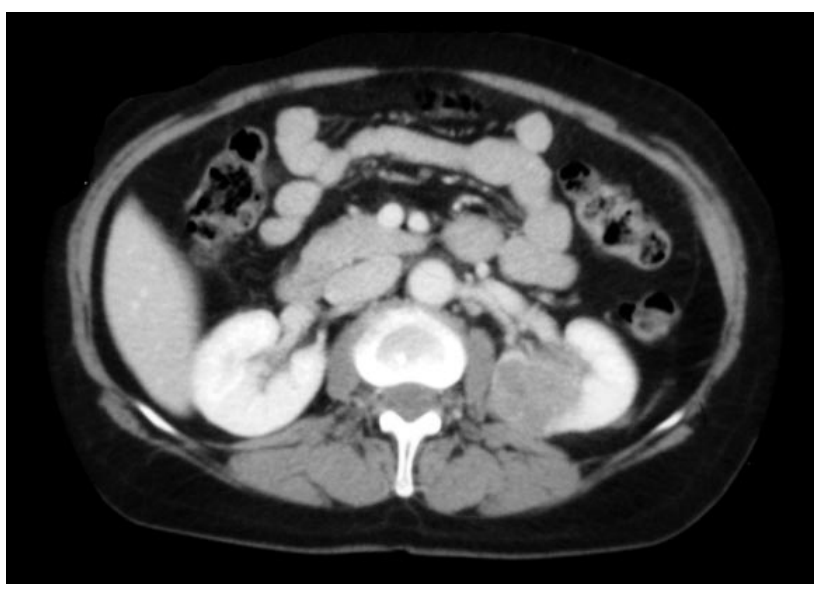

FIG URA 2. CT abdomen: masa renal izquierda de características mixtas 


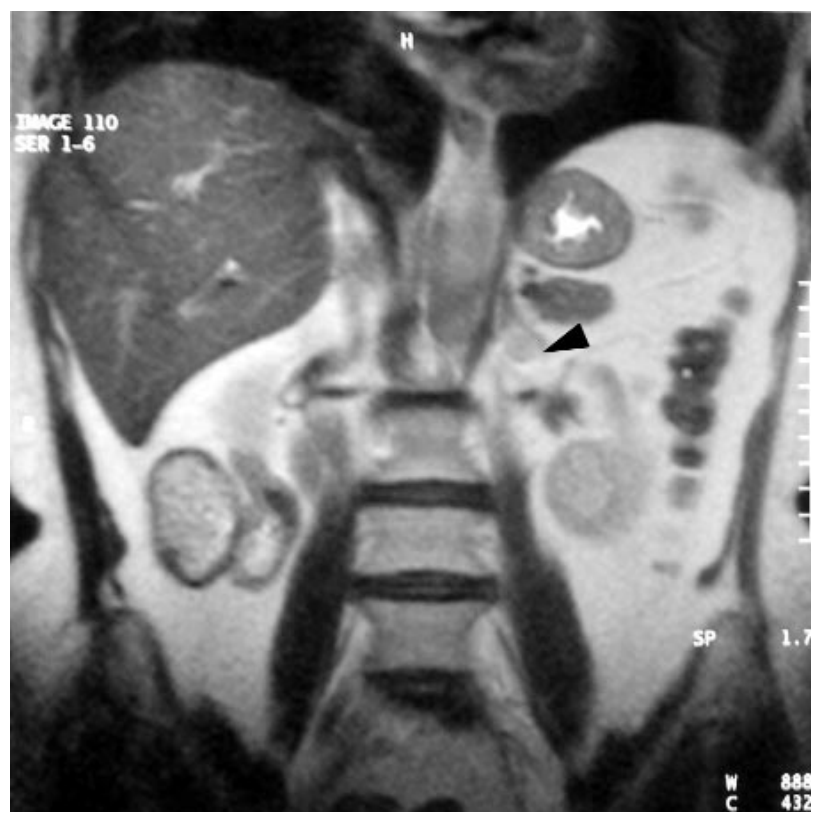

FIG URA 3. RMN : nódulo suprarrel izquierdo hipercaptante sin características adenomatosas

plasias antes citadas, pero no debe descartarse un tumor primario a menos que se practiquen las pruebas diagnósticas pertinentes.

Es llamativo en esta paciente la ausencia de crisis hipertensivas y el cortejo sintomático del feocromocitoma incluso durante la inducción anestésica, lo que nos hizo pensar que nos hallábamos ante una metástasis del carcinoma renal.

Las elevaciones tensionales que acontecen en el postoperatorio temprano de nuestra paciente reflejan cambios en la distribución de líquidos así como inestabilidad autonómica (3). En estos casos suele ser efectivo el empleo de diuréticos, beta bloqueantes y bloqueantes de los canales del calcio. En casos de refractariedad sería necesario tratamiento con fentolamina; la respuesta a este fármaco sugeriría que la exéresis del feocromocitoma no ha sido completa y sería necesaria una segunda intervención, previa determinación de catecolaminas en orina y sangre $(1,6)$.
**3. EGEA, J.; FERNÁNDEZ DEL BUSTO, E.; GONZÁLEZ DE ZÁRATE, J. y cols.: "Emergencia hipertensiva durante la cirugía de un carcinoma renal asociado a feocromocitoma no diagnosticado". Arch. Esp. Urol., 54: 707, 2001.

4. OZENZ, A.; RUACAN, S.; BAYKAL, A.: "Renal cell carcinoma and ipsilateral pheochromocytoma with neoplasm-to-neoplasm metastasis". J. Urol., 157: 1831, 1997.

5. SAGE, D.; HASSAN, T.; GRANDE-GOBURDHUN, J. y cols.: "Association of pheochromocitoma with homolateral clear cell renal carcinoma". Ann. Pathol., 11: 186, 1991.

6. LO, C.Y.; VAN HEERDEN, J.A.; SOREIDE, J.A. y cols.: "Adrenalectomy for metastasis disease to the adrenal glands". Br. J. Surgery, 83: 528, 1996.

Casos C línicos

Arch. Esp. Urol., 58, 4 (362-365), 2005

\section{MIELOUPOMA ADRENAL GIGANTE.}

M a Jesús Pareja M egía ${ }^{1}$, Rafael Barrero Canda $u^{2}$, $M$ anuel Medina Pérez y Juan Antonio Valero Puerta.

Servicios de Patología y Urología ${ }^{2}$. Hospital de la M erced. O suna. Sevilla. España

Resumen.- O BJETIVOS: Presentar un caso de mielolipoma gigante de la glándula suprarrenal.

M ÉTO DO S/ RESULTADO S: El mielolipoma gigante de la glándula suprarrenal es un tumor raro no funcionante de la corteza suprarrenal compuesto por tejido hematopoyético y adiposo que fue descubierto de forma accidental mediante TAC.

CON CLUSIO N ES: La conducta terapéutica es expectante en tumores pequeños y quirúrgica para los casos sintomáticos y tumores mayores de $4 \mathrm{~cm}$ en los que el riesgo de ruptura espontánea con hemorragia retroperitoneal es muy alto.

M a Jesús Pareja M ejía

Servicio de Patología. Hospital de la Merced

Avda. de la Constitución, 2

416400 suna. Sevilla. (España)

Trabajo recibido: 29 de noviembre de 2004

\section{BIBUOGRAFIA y LECTURAS}

RECOMENDADAS (*lectura de interés y

1. KLINGLER, H.C.; KLINGLER, P.J.; MARTIN, J.K. y cols.: "Pheochromocytoma". Urology, 57, 2001.

**2. JEFFRY, T.; MD CARLTON, C.; BARNETT, JR. y cols.: "Evaluation and surgical resection adrenal masses in patients with a history of extra-adrenal malignancy”. Surgery, 130: 1060, 2001. 